\title{
Oncostatin M promotes inflammation in IBD
}

New research published in Nature Medicine highlights an important role for the IL-6 family cytokine oncostatin $\mathrm{M}(\mathrm{OSM})$ in driving intestinal inflammation in IBD. Additionally, expression of OSM in intestinal biopsy samples from patients with IBD predicts response to anti-TNF agents.

Intestinal inflammation in IBD is associated with increased production of many pro-inflammatory cytokines, and anti-TNF antibody therapies represent a mainstay of IBD treatment. However, up to $40 \%$ of patients are nonresponsive to anti-TNF drugs, and many initially responsive patients develop treatment resistance. Two goals of current IBD research, therefore, are the development of therapeutics targeting pro-inflammatory mediators

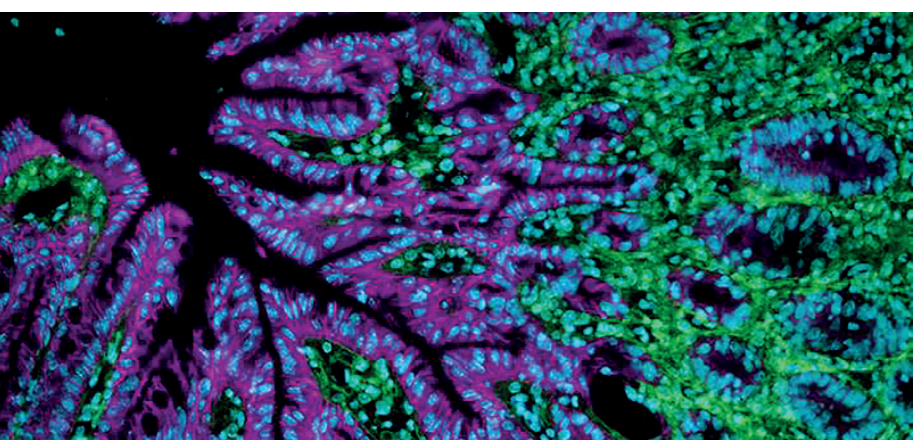

Immunofluorescence micrograph of inflamed mouse colonic mucosa, showing stromal cells (green), epithelial cells (magenta) and cell nuclei (cyan). Image courtesy of S. Bullers. other than TNF and the identification of biomarkers for nonresponsiveness to anti-TNF agents.

To identify cytokines associated with IBD pathogenesis, Nathaniel West and colleagues examined the mRNA expression of 64 candidate cytokines in intestinal mucosal biopsy samples from patients with active ulcerative colitis or Crohn's disease. Compared with tissue from healthy patients, only IL6, IL1A, ILIB and OSM were upregulated in both Crohn's disease and ulcerative colitis tissue samples. The least studied of these cytokines, OSM, was subsequently chosen as the focus of research.

In addition to validating the overexpression of intestinal OSM in other cohorts of patients with IBD, West and colleagues also found a strong association between increased OSM levels and resistance to anti-TNF therapy, suggesting that OSM represents a potential biomarker for anti-TNF nonresponsiveness. The researchers then analysed intestinal biopsy tissue to probe the role of the OSM pathway in IBD. "These experiments led to the surprising discovery that nonhaematopoietic, nonepithelial stromal cells are the major responders to OSM, based on their high expression of the OSM receptor (OSMR)," explains West. "Immunohistochemistry revealed that these stromal cells are present at very high numbers in the inflamed mucosa of patients with IBD." When treated with recombinant OSM, cultured human colon fibroblasts upregulated expression of IL6 and numerous chemokines.

The investigators also confirmed the presence of the OSM-stromal-cell axis in a mouse model of T-cell-mediated colitis that is nonresponsive to anti-TNF therapy. In functional experiments, colitis was attenuated by global knockout of Osm and by neutralization of OSM signalling using an injected soluble 'decoy' receptor.

"Several studies have shown an association between broad gene expression patterns and anti-TNF response, but ours is, to our knowledge, the first to reproducibly demonstrate the association of a single factor with treatment resistance in multiple independent cohorts," West observes. "Moving forward, we want to develop a better mechanistic understanding of how OSM promotes intestinal inflammation."

Hugh Thomas

ORIGINAL ARTICLE West, N. R. et al. Oncostatin $M$ drives intestinal inflammation and predicts response to tumor necrosis factor-neutralizing therapy in patients with inflammatory bowel disease. Nat. Med. http://dx.doi.org/10.1038/ nm.4307 (2017) 